\title{
A new error bound for linear complementarity problems for $B$-matrices
}

\author{
Chaoqian $\mathrm{Li}^{*}$, Mengting Gan, Shaorong Yang \\ School of Mathematics and Statistics, Yunnan University, Yunnan, P. R. China 650091
}

\begin{abstract}
A new error bound for the linear complementarity problem is given when the involved matrix is a $B$-matrix. It is shown that this bound is sharper than some previous bounds [C.Q. Li, Y.T. Li. Note on error bounds for linear complementarity problems for B-matrices, Applied Mathematics Letters, 57:108-113, 2016] and [C.Q. Li, Y.T. Li. Weakly chained diagonally dominant $B$-matrices and error bounds for linear complementarity problems, to appear in Numer. Algor.].
\end{abstract}

Keywords: Error bound, Linear complementarity problem, $B$-matrix

\section{Introduction}

Linear complementarity problem $\operatorname{LCP}(M, q)$ is to find a vector $x \in R^{n}$ such that

$$
x \geq 0, M x+q \geq 0,(M x+q)^{T} x=0,
$$

where $M=\left[m_{i j}\right] \in R^{n \times n}$ and $q \in R^{n}$. The $L C P(M, q)$ has various applications in the Nash equilibrium point of a bimatrix game, the contact problem and the free boundary problem for journal bearing, for details, see [4, 5, 17].

It is well-known that the $\operatorname{LCP}(M, q)$ has a unique solution for any $q \in R^{n}$ if and only if $M$ is a $P$-matrix [5]. Here, a matrix $M \in R^{n \times n}$ is called a $P$ matrix if all its principal minors are positive [6]. In [3], Chen and Xiang gave the following error bound of the $\operatorname{LCP}(M, q)$ when $M$ is a $P$-matrix:

$$
\left\|x-x^{*}\right\|_{\infty} \leq \max _{d \in[0,1]^{n}}\left\|(I-D+D M)^{-1}\right\|_{\infty}\|r(x)\|_{\infty},
$$

where $x^{*}$ is the solution of the $\operatorname{LCP}(M, q), r(x)=\min \{x, M x+q\}, D=\operatorname{diag}\left(d_{i}\right)$ with $0 \leq d_{i} \leq 1$, and the min operator $r(x)$ denotes the componentwise minimum of two vectors. If $M$ satisfies certain structure, then some bounds of

\footnotetext{
* Corresponding author.

Email address: lichaoqian@ynu.edu.cn (Chaoqian Li )
} 
$\max _{d \in[0,1]^{n}}\left\|(I-D+D M)^{-1}\right\|_{\infty}$ can be derived; for details, see [2, 7, 8, 10, 14] and references therein.

When $M$ is a $B$-matrix introduced by Peña in [6] as a subclass of $P$-matrices, García-Esnaola and Peña in [10] presented the following upper bound which is only related with the entries of $M$. Here a real matrix $M=\left[m_{i j}\right] \in R^{n \times n}$ is called a $B$-matrix [6] if for each $i \in N=\{1,2, \ldots, n\}$,

$$
\sum_{k \in N} m_{i k}>0 \text {, and } \frac{1}{n}\left(\sum_{k \in N} m_{i k}\right)>m_{i j} \text {, for any } j \in N \text { and } j \neq i \text {. }
$$

Theorem 1. 10, Theorem 2.2] Let $M=\left[m_{i j}\right] \in R^{n \times n}$ be a B-matrix with the form

$$
M=B^{+}+C,
$$

where

$$
B^{+}=\left[b_{i j}\right]=\left[\begin{array}{ccc}
m_{11}-r_{1}^{+} & \cdots & m_{1 n}-r_{1}^{+} \\
\vdots & & \vdots \\
m_{n 1}-r_{n}^{+} & \cdots & m_{n n}-r_{n}^{+}
\end{array}\right],
$$

and $r_{i}^{+}=\max \left\{0, m_{i j} \mid j \neq i\right\}$. Then

$$
\max _{d \in[0,1]^{n}}\left\|(I-D+D M)^{-1}\right\|_{\infty} \leq \frac{n-1}{\min \{\beta, 1\}},
$$

where $\beta=\min _{i \in N}\left\{\beta_{i}\right\}$ and $\beta_{i}=b_{i i}-\sum_{j \neq i}\left|b_{i j}\right|$.

As shown in [15], if the diagonal dominance of $B^{+}$is weak, i.e.,

$$
\beta=\min _{i \in N}\left\{\beta_{i}\right\}=\min _{i \in N}\left\{b_{i i}-\sum_{j \neq i}\left|b_{i j}\right|\right\}
$$

is small, then the bound (5) may be very large when $M$ is a $B$-matrix, which leads to that the estimate in (5) is always inaccurate, for details, see [15, 16]. To improve the bound (5), Li and Li [15] gave the following bound for $\max _{d \in[0,1]^{n}} \|(I-$ $D+D M)^{-1} \|_{\infty}$ when $M$ is a $B$-matrix.

Theorem 2. 15, Theorem 4] Let $M=\left[m_{i j}\right] \in R^{n \times n}$ be a B-matrix with the form $M=B^{+}+C$, where $B^{+}=\left[b_{i j}\right]$ is the matrix of (4). Then

$$
\begin{gathered}
\max _{d \in[0,1]^{n}}\left\|(I-D+D M)^{-1}\right\|_{\infty} \leq \sum_{i=1}^{n} \frac{n-1}{\min \left\{\bar{\beta}_{i}, 1\right\}} \prod_{j=1}^{i-1}\left(1+\frac{1}{\bar{\beta}_{j}} \sum_{k=j+1}^{n}\left|b_{j k}\right|\right), \\
\text { where } \bar{\beta}_{i}=b_{i i}-\sum_{j=i+1}^{n}\left|b_{i j}\right| l_{i}\left(B^{+}\right), l_{k}\left(B^{+}\right)=\max _{k \leq i \leq n}\left\{\frac{1}{\left|b_{i i}\right|} \sum_{\substack{j=k, j \neq i}}^{n}\left|b_{i j}\right|\right\} \text { and } \\
\prod_{j=1}^{i-1}\left(1+\frac{1}{\bar{\beta}_{j}} \sum_{k=j+1}^{n}\left|b_{j k}\right|\right)=1 \text { if } i=1 .
\end{gathered}
$$


Very recently, when $M$ is a weakly chained diagonally dominant $B$-matrix, Li and Li [16] gave a bound for $\max _{d \in[0,1]^{n}}\left\|(I-D+D M)^{-1}\right\|_{\infty}$. This bound holds true for the case that $M$ is a $B$-matrix because a $B$-matrix is a weakly chained diagonally dominant $B$-matrix [16].

Theorem 3. [16, Corollary 1] Let $M=\left[m_{i j}\right] \in R^{n \times n}$ be a B-matrix with the form $M=B^{+}+C$, where $B^{+}=\left[b_{i j}\right]$ is the matrix of (4). Then

$$
\max _{d \in[0,1]^{n}}\left\|(I-D+D M)^{-1}\right\|_{\infty} \leq \sum_{i=1}^{n}\left(\frac{n-1}{\min \left\{\tilde{\beta}_{i}, 1\right\}} \prod_{j=1}^{i-1} \frac{b_{j j}}{\tilde{\beta}_{j}}\right),
$$

where $\tilde{\beta}_{i}=b_{i i}-\sum_{j=i+1}^{n}\left|b_{i j}\right|>0$ and $\prod_{j=1}^{i-1} \frac{b_{j j}}{\tilde{\beta}_{j}}=1$ if $i=1$.

In this paper, we also focus on the error bound for the $\operatorname{LCP}(M, q)$, and gave a new bound for $\max _{d \in[0,1]^{n}}\left\|(I-D+D M)^{-1}\right\|_{\infty}$ when $M$ is a $B$-matrix. It is shown that this bound is more effective to estimate $\max _{d \in[0,1]^{n}}\left\|(I-D+D M)^{-1}\right\|_{\infty}$ than that in Theorem 1 and sharper than those in Theorems 2 and 3 ,

\section{Main results}

We first recall some definitions. A matrix $A=\left[a_{i j}\right] \in C^{n \times n}$ is called a strictly diagonally dominant $(S D D)$ matrix if for each $i \in N,\left|a_{i i}\right|>\sum_{\substack{j=1, j \neq i}}^{n}\left|a_{i j}\right|$. It is well-known that an $S D D$ matrix is nonsingular [1]. A matrix $A=\left[a_{i j}\right]$ is called a $Z$-matrix if $a_{i j} \leq 0$ for any $i \neq j$, and a nonsingular $M$-matrix if $A$ is a $Z$-matrix with $A^{-1}$ being nonnegative [1]. Next, several lemmas which will be used later are given.

Lemma 4. [18, Theorem 3.2] Let $A=\left[a_{i j}\right] \in R^{n \times n}$ be an SDD M-matrix. Then

$$
\left\|A^{-1}\right\|_{\infty} \leq \sum_{i=1}^{n}\left(\frac{1}{a_{i i}\left(1-u_{i}(A) l_{i}(A)\right)} \prod_{j=1}^{i-1} \frac{1}{1-u_{j}(A) l_{j}(A)}\right),
$$

where $u_{i}(A)=\frac{1}{\left|a_{i i}\right|} \sum_{j=i+1}^{n}\left|a_{i j}\right|, l_{k}(A)=\max _{k \leq i \leq n}\left\{\frac{1}{\left|a_{i i}\right|} \sum_{\substack{j=k, j \neq i}}^{n}\left|a_{i j}\right|\right\}$ and

$$
\prod_{j=1}^{i-1} \frac{1}{1-u_{j}(A) l_{j}(A)}=1 \text { if } i=1 \text {. }
$$

Lemma 5. [15, Lemma 3] Let $\gamma>0$ and $\eta \geq 0$. Then for any $x \in[0,1]$,

$$
\frac{1}{1-x+\gamma x} \leq \frac{1}{\min \{\gamma, 1\}}
$$


and

$$
\frac{\eta x}{1-x+\gamma x} \leq \frac{\eta}{\gamma}
$$

Lemma 6. [16, Lemma 5] Let $A=\left[a_{i j}\right] \in R^{n \times n}$ with

$$
a_{i i}>\sum_{j=i+1}^{n}\left|a_{i j}\right| \text { for each } i \in N \text {. }
$$

Then for any $x_{i} \in[0,1], i \in N$,

$$
\frac{1-x_{i}+a_{i i} x_{i}}{1-x_{i}+a_{i i} x_{i}-\sum_{j=i+1}^{n}\left|a_{i j}\right| x_{i}} \leq \frac{a_{i i}}{a_{i i}-\sum_{j=i+1}^{n}\left|a_{i j}\right|} .
$$

The main result is given as follows.

Theorem 7. Let $M=\left[m_{i j}\right] \in R^{n \times n}$ be a B-matrix with the form $M=B^{+}+C$, where $B^{+}=\left[b_{i j}\right]$ is the matrix of (4). Then

$$
\max _{d \in[0,1]^{n}}\left\|(I-D+D M)^{-1}\right\|_{\infty} \leq \sum_{i=1}^{n} \frac{n-1}{\min \left\{\bar{\beta}_{i}, 1\right\}} \prod_{j=1}^{i-1} \frac{b_{j j}}{\bar{\beta}_{j}}
$$

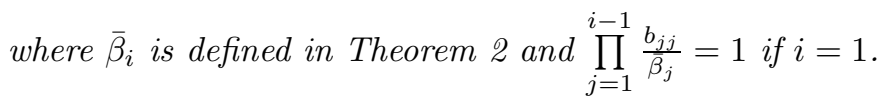

Proof. Let $M_{D}=I-D+D M$. Then

$$
M_{D}=I-D+D M=I-D+D\left(B^{+}+C\right)=B_{D}^{+}+C_{D},
$$

where $B_{D}^{+}=I-D+D B^{+}=\left[b_{i j}\right]$. Similar to the proof of Theorem 2.2 in [10], we can obtain that $B_{D}^{+}$is an $S D D M$-matrix with positive diagonal elements and $C_{D}=D C$, and that

$$
\left\|M_{D}^{-1}\right\|_{\infty} \leq\left\|\left(I+\left(B_{D}^{+}\right)^{-1} C_{D}\right)^{-1}\right\|_{\infty}\left\|\left(B_{D}^{+}\right)^{-1}\right\|_{\infty} \leq(n-1)\left\|\left(B_{D}^{+}\right)^{-1}\right\|_{\infty} .
$$

By Lemma 4 ,

$\left\|\left(B_{D}^{+}\right)^{-1}\right\|_{\infty} \leq \sum_{i=1}^{n}\left(\frac{1}{\left(1-d_{i}+b_{i i} d_{i}\right)\left(1-u_{i}\left(B_{D}^{+}\right) l_{i}\left(B_{D}^{+}\right)\right)} \prod_{j=1}^{i-1} \frac{1}{1-u_{j}\left(B_{D}^{+}\right) l_{j}\left(B_{D}^{+}\right)}\right)$,

where

$$
u_{i}\left(B_{D}^{+}\right)=\frac{\sum_{j=i+1}^{n}\left|b_{i j}\right| d_{i}}{1-d_{i}+b_{i i} d_{i}} \text {, and } l_{k}\left(B_{D}^{+}\right)=\max _{k \leq i \leq n}\left\{\frac{\sum_{\substack{j=k \\ j \neq i}}^{n}\left|b_{i j}\right| d_{i}}{1-d_{i}+b_{i i} d_{i}}\right\} .
$$


By Lemma 5, we can easily get that for each $k \in N$,

$$
l_{k}\left(B_{D}^{+}\right) \leq \max _{k \leq i \leq n}\left\{\frac{1}{b_{i i}} \sum_{\substack{j=k, j \neq i}}^{n}\left|b_{i j}\right|\right\}=l_{k}\left(B^{+}\right)<1,
$$

and that for each $i \in N$,

$$
\begin{aligned}
\frac{1}{\left(1-d_{i}+b_{i i} d_{i}\right)\left(1-u_{i}\left(B_{D}^{+}\right) l_{i}\left(B_{D}^{+}\right)\right)} & =\frac{1}{1-d_{i}+b_{i i} d_{i}-\sum_{j=i+1}^{n}\left|b_{i j}\right| d_{i} l_{i}\left(B_{D}^{+}\right)} \\
& \leq \frac{1}{\min \left\{b_{i i}-\sum_{j=i+1}^{n}\left|b_{i j}\right| l_{i}\left(B^{+}\right), 1\right\}} \\
& =\frac{1}{\min \left\{\bar{\beta}_{i}, 1\right\}} .
\end{aligned}
$$

Furthermore, by Lemma 6

$$
\frac{1}{1-u_{i}\left(B_{D}^{+}\right) l_{i}\left(B_{D}^{+}\right)}=\frac{1-d_{i}+b_{i i} d_{i}}{1-d_{i}+b_{i i} d_{i}-\sum_{j=i+1}^{n}\left|b_{i j}\right| d_{i} l_{i}\left(B_{D}^{+}\right)} \leq \frac{b_{i i}}{\bar{\beta}_{i}}
$$

By (11), (11), (12) and (13), we have

$$
\left\|\left(B_{D}^{+}\right)^{-1}\right\|_{\infty} \leq \frac{1}{\min \left\{\bar{\beta}_{1}, 1\right\}}+\sum_{i=2}^{n} \frac{1}{\min \left\{\bar{\beta}_{i}, 1\right\}} \prod_{j=1}^{i-1} \frac{b_{j j}}{\bar{\beta}_{j}} .
$$

The conclusion follows from (10) and (14).

The comparisons of the bounds in Theorems 2, 3 and 7 are established as follows.

Theorem 8. Let $M=\left[m_{i j}\right] \in R^{n \times n}$ be a B-matrix with the form $M=B^{+}+C$, where $B^{+}=\left[b_{i j}\right]$ is the matrix of (4). Let $\bar{\beta}_{i}$ and $\tilde{\beta}_{i}$ be defined in Theorems 0 and 3 respectively. Then

$$
\begin{aligned}
\sum_{i=1}^{n} \frac{n-1}{\min \left\{\bar{\beta}_{i}, 1\right\}} \prod_{j=1}^{i-1} \frac{b_{j j}}{\bar{\beta}_{j}} & \leq \sum_{i=1}^{n} \frac{n-1}{\min \left\{\bar{\beta}_{i}, 1\right\}} \prod_{j=1}^{i-1}\left(1+\frac{1}{\bar{\beta}_{j}} \sum_{k=j+1}^{n}\left|b_{j k}\right|\right) \\
& \leq \sum_{i=1}^{n}\left(\frac{n-1}{\min \left\{\tilde{\beta}_{i}, 1\right\}} \prod_{j=1}^{i-1} \frac{b_{j j}}{\tilde{\beta}_{j}}\right) .
\end{aligned}
$$

Proof. Note that

$$
\tilde{\beta}_{i}=b_{i i}-\sum_{j=i+1}^{n}\left|b_{i j}\right|, \bar{\beta}_{i}=b_{i i}-\sum_{j=i+1}^{n}\left|b_{i j}\right| l_{i}\left(B^{+}\right)
$$


and $l_{k}\left(B^{+}\right)=\max _{k \leq i \leq n}\left\{\frac{1}{\left|b_{i i}\right|} \sum_{\substack{j=k, j \neq i}}^{n}\left|b_{i j}\right|\right\}<1$. Hence, for each $i \in N, \tilde{\beta}_{i} \leq \bar{\beta}_{i}$ and

$$
\frac{1}{\min \left\{\tilde{\beta}_{i}, 1\right\}} \geq \frac{1}{\min \left\{\bar{\beta}_{i}, 1\right\}} .
$$

Meantime, for $j=1,2, \ldots, n-1$,

$$
1+\frac{1}{\bar{\beta}_{j}} \sum_{k=j+1}^{n}\left|b_{j k}\right| \leq 1+\frac{1}{\tilde{\beta}_{j}} \sum_{k=j+1}^{n}\left|b_{j k}\right|=\frac{1}{\tilde{\beta}_{j}}\left(\tilde{\beta}_{j}+\sum_{k=j+1}^{n}\left|b_{j k}\right|\right)=\frac{b_{j j}}{\tilde{\beta}_{j}} .
$$

By (15) and (16), we have

$$
\sum_{i=1}^{n} \frac{n-1}{\min \left\{\bar{\beta}_{i}, 1\right\}} \prod_{j=1}^{i-1}\left(1+\frac{1}{\bar{\beta}_{j}} \sum_{k=j+1}^{n}\left|b_{j k}\right|\right) \leq \sum_{i=1}^{n}\left(\frac{n-1}{\min \left\{\tilde{\beta}_{i}, 1\right\}} \prod_{j=1}^{i-1} \frac{b_{j j}}{\tilde{\beta}_{j}}\right) .
$$

Moreover, for $j=1,2, \ldots, n-1$,

$$
\begin{aligned}
\frac{b_{j j}}{\bar{\beta}_{j}} & =\prod_{j=1}^{i-1} \frac{b_{j j}-\sum_{k=j+1}^{n}\left|b_{j k}\right| l_{j}\left(B^{+}\right)+\sum_{k=j+1}^{n}\left|b_{j k}\right| l_{j}\left(B^{+}\right)}{\bar{\beta}_{j}} \\
& \left.=\frac{\bar{\beta}_{j}+\sum_{k=j+1}^{n}\left|b_{j k}\right| l_{j}\left(B^{+}\right)}{\bar{\beta}_{j}}\right) \\
& =\left(1+\frac{\sum_{k=j+1}^{n}\left|b_{j k}\right| l_{j}\left(B^{+}\right)}{\bar{\beta}_{j}}\right) \\
& \leq\left(1+\frac{\sum_{k=j+1}^{n}\left|b_{j k}\right|}{\bar{\beta}_{j}}\right)
\end{aligned}
$$

this implies

$$
\sum_{i=1}^{n} \frac{n-1}{\min \left\{\bar{\beta}_{i}, 1\right\}} \prod_{j=1}^{i-1} \frac{b_{j j}}{\bar{\beta}_{j}} \leq \sum_{i=1}^{n} \frac{n-1}{\min \left\{\bar{\beta}_{i}, 1\right\}} \prod_{j=1}^{i-1}\left(1+\frac{1}{\bar{\beta}_{j}} \sum_{k=j+1}^{n}\left|b_{j k}\right|\right) .
$$

The conclusion follows from (17) and (18).

Example 1. Consider the family of $B$-matrices in [15]:

$$
M_{k}=\left[\begin{array}{cccc}
1.5 & 0.5 & 0.4 & 0.5 \\
-0.1 & 1.7 & 0.7 & 0.6 \\
0.8 & -0.1 \frac{k}{k+1} & 1.8 & 0.7 \\
0 & 0.7 & 0.8 & 1.8
\end{array}\right]
$$


where $k \geq 1$. Then $M_{k}=B_{k}^{+}+C_{k}$, where

$$
B_{k}^{+}=\left[\begin{array}{cccc}
1 & 0 & -0.1 & 0 \\
-0.8 & 1 & 0 & -0.1 \\
0 & -0.1 \frac{k}{k+1}-0.8 & 1 & -0.1 \\
-0.8 & -0.1 & 0 & 1
\end{array}\right] .
$$

By Theorem 10(Theorem 2.2 in [10]), we have

$$
\max _{d \in[0,1]^{4}}\left\|(I-D+D M)^{-1}\right\|_{\infty} \leq \frac{4-1}{\min \{\beta, 1\}}=30(k+1) .
$$

It is obvious that

$$
30(k+1) \rightarrow+\infty \text {, when } k \rightarrow+\infty .
$$

By Theorem 3, we have

$$
\max _{d \in[0,1]^{4}}\left\|(I-D+D M)^{-1}\right\|_{\infty} \leq \sum_{i=1}^{4}\left(\frac{3}{\min \left\{\tilde{\beta}_{i}, 1\right\}} \prod_{j=1}^{i-1} \frac{b_{j j}}{\tilde{\beta}_{j}}\right) \approx 15.2675 .
$$

By Theorem 2, we have

$$
\max _{d \in[0,1]^{4}}\left\|\left(I-D+D M_{k}\right)^{-1}\right\|_{\infty} \leq \frac{2.97(90 k+91)(190 k+192)+6.24(100 k+101)^{2}}{0.99(90 k+91)^{2}},
$$

and

$$
\frac{2.97(90 k+91)(190 k+192)+6.24(100 k+101)^{2}}{0.99(90 k+91)^{2}}<15.2675, \text { for any } k \geq 1 .
$$

By Theorem 7, we have

$$
\max _{d \in[0,1]^{4}}\left\|\left(I-D+D M_{k}\right)^{-1}\right\|_{\infty} \leq \frac{2.97(90 k+91)(190 k+191)+5.97(100 k+100)^{2}}{0.99(90 k+91)^{2}},
$$

and

$$
\begin{aligned}
& \frac{2.97(90 k+91)(190 k+191)+5.97(100 k+100)^{2}}{0.99(90 k+91)^{2}} \\
< & \frac{2.97(90 k+91)(190 k+192)+6.24(100 k+101)^{2}}{0.99(90 k+91)^{2}} .
\end{aligned}
$$

In particular, when $k=1$,

$$
\frac{2.97(90 k+91)(190 k+191)+5.97(100 k+100)^{2}}{0.99(90 k+91)^{2}} \approx 13.6777
$$

and

$$
\frac{2.97(90 k+91)(190 k+192)+6.24(100 k+101)^{2}}{0.99(90 k+91)^{2}} \approx 14.1044
$$


When $k=2$,

$$
\frac{2.97(90 k+91)(190 k+191)+5.97(100 k+100)^{2}}{0.99(90 k+91)^{2}} \approx 13.7110
$$

and

$$
\frac{2.97(90 k+91)(190 k+192)+6.24(100 k+101)^{2}}{0.99(90 k+91)^{2}} \approx 14.1079 .
$$

In these two cases, the bounds in (5) are equal to $60(k=1)$ and $90(k=2)$ respectively. This example shows that the bound in Theorem 7 is sharper than those in Theorems 1, 2 and 3 .

\section{Conclusions}

In this paper, we give a new bound for $\max _{d \in[0,1]^{n}}\left\|(I-D+D M)^{-1}\right\|_{\infty}$ when $M$ is a $B$-matrix, which improves the bounds obtained in [10, 15, 16].

\section{Acknowledgements}

This work is supported by National Natural Science Foundations of China (11361074), Natural Science Foundations of Yunnan Province (2013FD002) and IRTSTYN.

\section{References}

[1] A. Berman, R.J. Plemmons. Nonnegative Matrix in the Mathematical Sciences. SIAM Publisher, Philadelphia, 1994.

[2] T.T. Chen, W. Li, X. Wu, S. Vong. Error bounds for linear complementarity problems of MB-matrices. Numer. Algor., Doi:10.1007/s11075-014-9950-9.

[3] X.J. Chen and S.H. Xiang, Computation of error bounds for P-matrix linear complementarity problem, Mathematical Programming, 106:513-525, 2006.

[4] X.J. Chen and S.H. Xiang. Perturbation bounds of $P$-matrix linear complementarity problems. SIAM J. Optim., 18:1250-1265, 2007.

[5] R.W. Cottle, J.S. Pang, R.E. Stone. The Linear Complementarity Problem. Academic Press, San Diego, 1992.

[6] J.M. Peña. A class of $P$-matrices with applications to the localization of the eigenvalues of a real matrix. SIAM J. Matrix Anal. Appl., 22:1027-1037, 2001.

[7] P.F. Dai. Error bounds for linear complementarity problems of $D B$ matrices. Linear Algebra Appl., 434:830-840, 2011. 
[8] P.F. Dai, Y.T. Li and C.J. Lu. Error bounds for linear complementarity problems for SB-matrices. Numer. Algor., 61:121-139, 2012.

[9] P.F. Dai, C.J. Lu and Y.T. Li. New error bounds for the linear complementarity problem with an SB-matrix. Numer. Algor., 64(4):741-757, 2013.

[10] M. García-Esnaola and J.M. Peña. Error bounds for linear complementarity problems for B-matrices. Appl. Math. Lett., 22:1071-1075, 2009.

[11] M. García-Esnaola and J.M. Peña. Error bounds for the linear complementarity problem with a $\Sigma$-SDD matrix. Linear Algebra and its Applications, 438(3): 1339-1346, 2013.

[12] M. García-Esnaola and J.M. Peña. Error bounds for linear complementarity problems involving $B^{S}$-matrices. Appl. Math. Lett., 25(10):1379-1383, 2012.

[13] M. García-Esnaola and J.M. Peña. Error bounds for linear complementarity problems of Nekrasov matrices. Numer. Algor., 67:655-667, 2014.

[14] M. García-Esnaola and J.M. Peña. B-Nekrasov matrices and error bounds for linear complementarity problems, Numer. Algor., DOI:10.1007/s11075015-0054-y.

[15] C.Q. Li, Y.T. Li. Note on error bounds for linear complementarity problems for B-matrices, Applied Mathematics Letters, 57:108-113, 2016.

[16] C.Q. Li, Y.T. Li. Weakly chained diagonally dominant B-matrices and error bounds for linear complementarity problems, to appear in Numer. Algor..

[17] K.G. Murty. Linear Complementarity, Linear and Nonlinear Programming. Heldermann Verlag, Berlin, 1988.

[18] P. Wang. An upper bound for $\left\|A^{-1}\right\|_{\infty}$ of strictly diagonally dominant M-matrices. Linear Algebra Appl., 431:511-517, 2009. 